\title{
Tunable multiband terahertz metamaterials using a reconfigurable electric split-ring resonator array
}

\begin{abstract}
Fusheng $\mathrm{Ma}^{1}$, Yu-Sheng Lin ${ }^{1}$, Xinhai Zhang ${ }^{2}$ and Chengkuo Lee ${ }^{1}$
We demonstrate micromachined reconfigurable metamaterials working at multiple frequencies simultaneously in the terahertz range. The proposed metamaterial structures can be structurally reconfigured by employing flexible microelectromechanical system-based cantilevers in the resonators, which are designed to deform out of plane under an external stimulus. The proposed metamaterial structures provide not only multiband resonance frequency operation but also polarization-dependent tunability. Three kinds of metamaterials are investigated as electric split-ring resonator (eSRR) arrays with different positions of the split. By moving the position of the split away from the resonator's center, the eSRR exhibits anisotropy, with the dipole resonance splitting into two resonances. The dipole-dipole coupling strength can be continuously adjusted, which enables the electromagnetic response to be tailored by adjusting the direct current (DC) voltage between the released cantilevers and the silicon substrate. The observed tunability of the eSRRs is found to be dependent on the polarization of the incident terahertz wave. This polarization-dependent tunability is demonstrated by both experimental measurements and electromagnetic simulations.
\end{abstract}

Light: Science \& Applications (2014) 3, e171; doi:10.1038/lsa.2014.52; published online 23 May 2014

Keywords: MEMS; reconfigurable metamaterials; split-ring resonator; terahertz; tunability

\section{INTRODUCTION}

Electromagnetic waves in the terahertz $(\mathrm{THz})$ frequency range have received tremendous attention due to their various advantages. Much research has been carried out to characterize the interactions of these waves with matter, for which potential applications include medical imaging, security screening and non-destructive evaluation. However, there are still several restrictions that limit the full exploitation of fruitful applications covering the $\mathrm{THz}$ region due to the lack of an appropriate response at these frequencies for many naturally existing materials. ${ }^{1-4}$ As artificially structured electromagnetic materials, metamaterials have been extensively investigated for the possibility of creating novel electromagnetic properties that are not available in natural materials, such as a negative refractive index, superlensing and cloaking. ${ }^{1-4}$ The electric permittivity and magnetic permeability of metamaterials can be designed for desired specifications and can be scaled to operate over nearly the entire electromagnetic spectrum. These artificial materials could find potential applications in the development of novel $\mathrm{THz}$ devices, which is traditionally difficult to achieve due to the absence of suitable functional sources and detectors. ${ }^{5-9}$ Due to the limitations of fabrication and characterization technology, ${ }^{10-12}$ investigations of metamaterial-based devices were initially implemented in the microwave frequency range. Metamaterials that operate in the $\mathrm{THz}$ range have attracted intense interest along with the advancement of fabrication technologies. ${ }^{7,13-15}$ Inspired by the realization of tunability, the research on metamaterials has been extended from the structural design of a specific electromagnetic response to reconfigurable $\mathrm{THz}$ metamaterial devices with a broad working bandwidth. ${ }^{16,17}$ To realize tunability in metamaterials, various approaches have been demonstrated to vary effective electromagnetic properties via laser light illumination, ${ }^{17,18}$ external magnetostatic fields, ${ }^{19}$ external bias voltages, ${ }^{16,20,21}$ electrical or thermal effects in liquid crystals ${ }^{22-28}$ and nonlinear effects of resonators or subtrates. ${ }^{16,17,29-36}$ Alternatively, structural reconfiguration is a novel and straightforward method for controlling the electromagnetic properties, including the amplitude, polarization and directionality of the metamaterial structures. The properties of metamaterials can be directly modified by reconfiguring the fundamental building block of the metamaterial. Using microelectromechanical system (MEMS) technology, tunable metamaterials have been demonstrated. ${ }^{37-41}$ The geometric dimensions of metamaterial structures designed for $\mathrm{THz}$ operations are on the order of tens of microns or even smaller for higher frequencies. Therefore, geometrically altered metamaterial unit cells based on MEMS-actuated structures are promising for unprecedented tunability and may overcome the limitations of the constituent materials. ${ }^{38,42-47}$

In addition, dual-band and multiband metamaterials with negative permeability property have recently been investigated. ${ }^{30,48-50}$ Metamaterials with multiple-resonance excitation provide more potential for designing complex and powerful optical devices. In this work, we present multiband metamaterials using a MEMS-based alternative electric split-ring resonator (eSRR). Differing from MEMS-based metamaterial structures using an in-plane comb drive actuator produced by deep reactive ion etching, ${ }^{40,42}$ we incorporated

${ }^{1}$ Department of Electrical and Computer Engineering, National University of Singapore, Singapore 117576, Singapore and ${ }^{2}$ Institute of Materials Research and Engineering, A*STAR, Singapore 117602 , Singapore

Correspondence: Dr C Lee, Department of Electrical and Computer Engineering, National University of Singapore, Singapore 117576, Singapore

E-mail: elelc@nus.edu.sg

Received 29 May 2013; revised 16 December 2013; accepted 15 February 2014 
MEMS-based bimorph cantilevers into the unit cell of the eSRR, where such bimorph cantilevers deflect toward the out-of-plane direction. The proposed MEMS-based metamaterial structures are geometrically reconfigurable and can be easily scaled by electrically reconfiguring the metamaterial unit cells. Furthermore, the upward-bending bimorph cantilevers can result in out-of-plane tunable metamaterials without the need for complicated fabrication processes. ${ }^{51}$ Using the electrostatic actuation mechanism, the bending degree of the MEMS-based bimorph cantilevers can be dynamically adjusted to achieve a tunable electromagnetic response in eSRR metamaterials, i.e., resulting in not only multiband resonance frequency operation but also polarizationdependent tunability. Multiband properties are desirable in this phenomenon for creating multifunctional devices for several potential applications. For instance, our proposed dynamically tunable metamaterials are highly desirable for $\mathrm{THz}$ applications in high-sensitivity sensing, tunable filtering, switching, frequency-selective detection, multi-spectral imaging, etc.

\section{MATERIALS AND METHODS}

\section{Schematic}

Figure 1a shows the eSRRs used in the proposed metamaterial structures. The classical eSRR structure shown in Figure 1a is considered as a composite of two rings with a common 'split'. An inductive effect is attributed to the current induced in the eSRR rings as well as a capacitive effect across the 'split'. ${ }^{7}$ Therefore, the eSRR can be qualitatively described in terms of its equivalent circuit. ${ }^{7}$ A capacitor-like 'split' structure regarded with a capacitance $(C)$ couples to the electric field, and the two rings of the eSRR provide an inductance $(L)$ to the circuit, so the resonant frequency of the circuit model is $\omega=\sqrt{2 / L C}$. Hence, the resonance frequency of the eSRR can be changed either by varying the dimensions of the rings to alter the qualitative inductance or by varying the split to adjust the qualitative capacitance. The dimensional parameters of the eSRR are shown in Figure 1a with length $L=80 \mu \mathrm{m}$, beam width $W=4 \mu \mathrm{m}$, split wall width $G=20 \mu \mathrm{m}$ and a split gap width of $4 \mu \mathrm{m}$. The position of the split is represented by the length lof the split gap-bearing side of the eSRRs. In this study, two new kinds of eSRRs are proposed by changing the position of the 'split' from the eSRR's center to its edge. These two modified eSRRs are expected to exhibit different transmission characteristics compared to the classical eSRR. For brevity, the eSRRs with different split positions are referred to as eSRR01, eSRR02 and eSRR03 for $l=30,48$ and $67 \mu \mathrm{m}$, respectively. The electromagnetic tunability of these eSRRs is achieved by incorporating MEMS-based bimorph cantilevers with an adjustable height $h$, as shown in Figure 1b. The capacitance of the eSRR structure depends on the height of the cantilever. Various actuation methods can be utilized to adjust the position of the cantilever. Here, electrostatic actuation is investigated for actuating the bimorph cantilever. Figure 1b-1d shows the unit cell of the eSRRs, with the released bimorph cantilevers bending upwards due to the tensile stress of the cantilever after they are released from the substrate. A two-dimensional eSRR array with a period of $120 \mu \mathrm{m}$ is formed by electrically connecting individual eSRRs using conducting metal lines. As an example, a chained eSRR02 array is schematically depicted in Figure 1e. The metal lines connecting the individual eSRRs provide electrical connectivity for performing electrostatic operations throughout the entire eSRR array. Depending on whether the incident electric field is perpendicular or parallel to the connecting metal lines, the incident $\mathrm{THz}$ wave is of extraordinary polarization (e-polarization) or ordinary polarization (o-polarization), ${ }^{16}$ as shown in Figure 1e.

\section{Fabrication}

The proposed structurally reconfigurable metamaterials, consisting of a two-dimensional square-lattice array of eSRRs with a unit cell of $120 \times 120 \mu^{2}$, are fabricated by a Complementary Metal-Oxide Semiconductor (CMOS)-compatible process, followed by a micromachining release step using vapor hydrofluoric acid (VHF), as shown in Figure 2. During the fabrication process, two masks are used to enable structure anchoring and eSRR array patterning. The fabrication starts with low-pressure chemical vapor deposition of a 100-nm-thick silicon oxide $\left(\mathrm{SiO}_{2}\right)$ on a standard p-doped silicon wafer with a resistivity of $0.05 \sim 3 \Omega \mathrm{m}$. The $\mathrm{SiO}_{2}$ layer is then etched with reactive ion etching to anchor the structure using the first mask, as shown in Figure 2b. Secondly, a 20-nm-thick aluminum oxide $\left(\mathrm{Al}_{2} \mathrm{O}_{3}\right)$ layer and a 500nm-thick aluminum $(\mathrm{Al})$ layer are deposited using atomic layer deposition and physical vapor deposition, respectively. Then, the $\mathrm{Al} / \mathrm{Al}_{2} \mathrm{O}_{3}$ bilayer is patterned with the second mask for the metamaterial pattern, as shown in Figure 2e. After the CMOS fabrication process, the fabricated eSRR arrays are released using VHF. The cantilevers incorporated into the eSRRs bend upward due to the residual tensile stress of the $\mathrm{Al} / \mathrm{Al}_{2} \mathrm{O}_{3}$ bilayer, as shown in Figure $2 \mathrm{f}$. An eSRR array with an overall size of approximately $1 \times 1 \mathrm{~cm}^{2}$ ( $83 \times 83$ unit cells) is fabricated on a standard silicon wafer. Figure $3 a-3 c$ shows scanning electron microscopy images of the unit cell of the unreleased eSRR01,

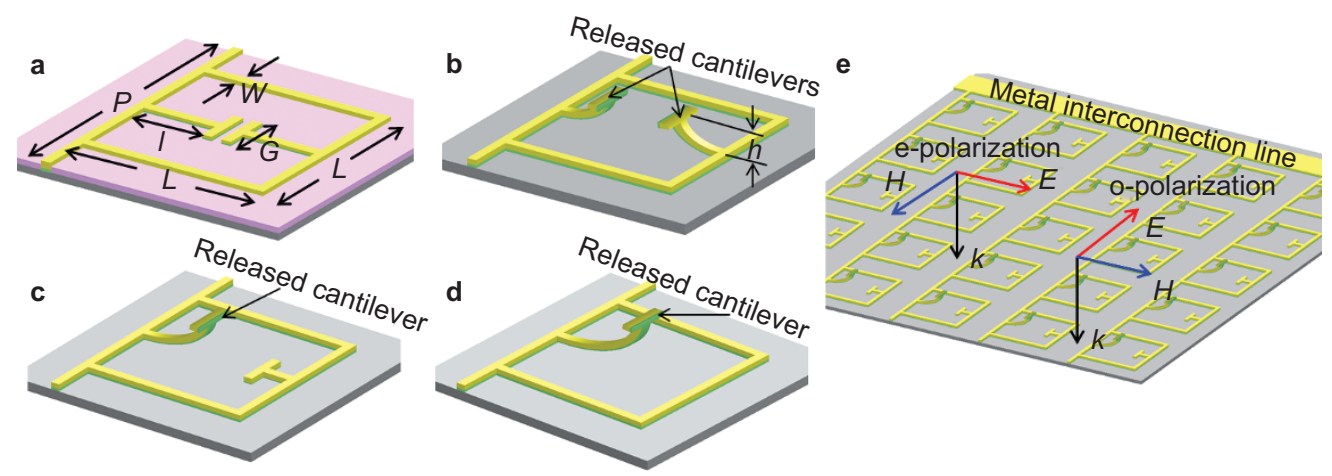

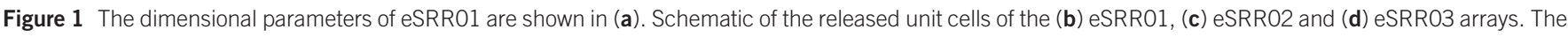

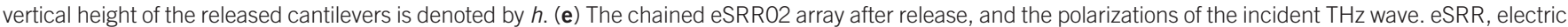
split-ring resonator; $\mathrm{THz}$, terahertz. 

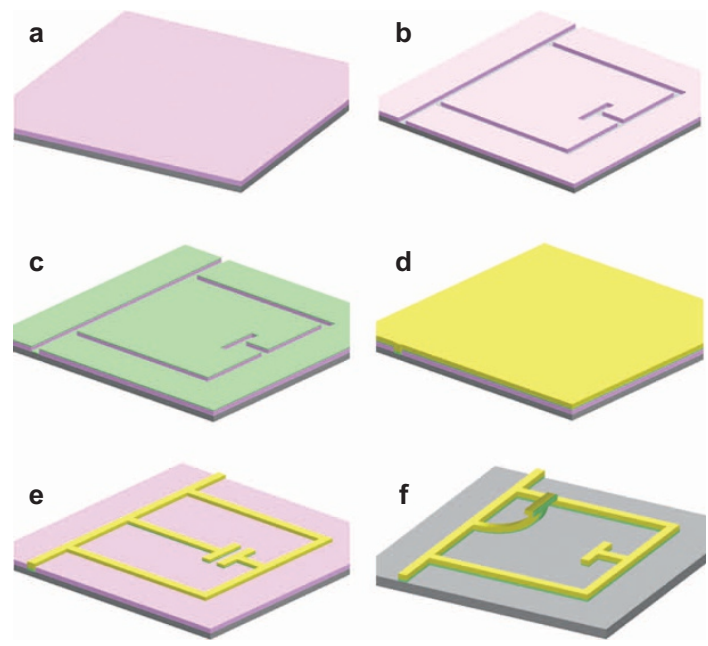

Figure 2 Fabrication process of the eSRRs: (a) deposition of $\mathrm{SiO}_{2}$; (b) RIE for anchoring; (c) deposition of the $\mathrm{Al}_{2} \mathrm{O}_{3}$ layer; (d) deposition of the Al layer; (e) metamaterial structure patterning; (f) releasing using VHF. eSRR, electric splitring resonator; RIE, reactive ion etching; VHF, vapor hydrofluoric acid.

eSRR02 and eSRR03, respectively. Scanning electron microscopy images of the unit cell of the released eSRR arrays indicate that the cantilevers are successfully released, as shown in Figure $3 \mathrm{~d}-3 \mathrm{f}$.

\section{Characterization}

The electromagnetic responses of the eSRR metamaterials were characterized by measuring the transmission spectrum of the metamaterials using $\mathrm{THz}$ time domain spectroscopy (TeraView TPS 3000; Teraview Ltd., Cambridge, UK) in transmission mode. The experimental measurements were performed at room temperature in a
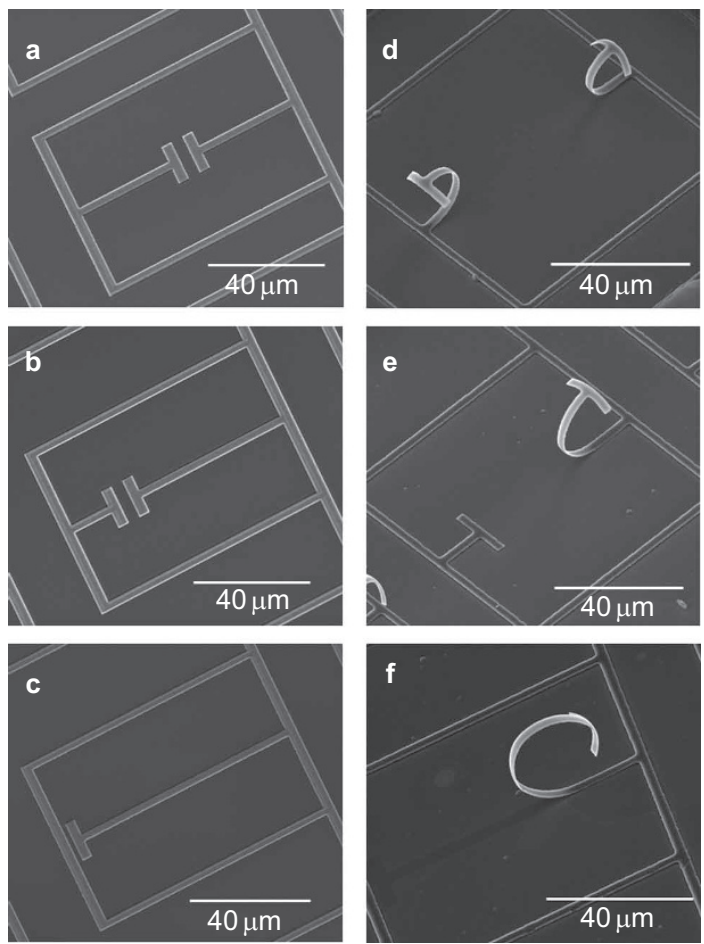

Figure 3 Scanning electron microscopy images of unit cells of the eSRRO1, eSRR02 and eSRR03 arrays with T-shaped cantilevers: (a-c) non-released and $(\mathbf{d}-\mathbf{f})$ released, respectively. eSRR, electric split-ring resonator. nitrogen-filled chamber. By recording the time-varying electric field of the impulsive $\mathrm{THz}$ radiation, and the amplitude and phase of the electric field spectra were directly obtained by performing a Fourier analysis. Transmission spectra were recorded for two orthogonal polarization states corresponding to the e- and o-polarization incidence. To experimentally verify the tunability of the proposed metamaterials, electrostatic actuation was realized by applying a direct current (DC) voltage between the interconnection lines merged at the edges of the array and the Si substrate.

\section{Operation principle and mechanical simulation}

The actuation of the cantilevers is based on the electrostatic attractive force between the released cantilevers and the grounded substrate when a DC bias voltage is applied. The operation principle of the device is illustrated in Figure 4a. In the initial state where the applied voltage $V=0$, the bilayer cantilevers bend upward resulting from the initial stress induced in the $\mathrm{Al}$ and $\mathrm{Al}_{2} \mathrm{O}_{3}$ layers during the VHF release progress. The mismatch in the thermal expansion coefficients of $\mathrm{Al}$ and $\mathrm{Al}_{2} \mathrm{O}_{3}$ causes out-of-plane deformations of the bilayer cantilevers. The cantilever curves upward because the $\mathrm{Al}_{2} \mathrm{O}_{3}$ is under compressive residual stress, while the $\mathrm{Al}$ film is under tensile residual stress. When the applied DC voltage $V$ increases, the bilayer cantilevers are attracted from the initial position downwards to the substrate by the electrostatic force. At this balanced intermediate position, the deformation of the cantilevers is determined by the balance between the electrostatic force and the cantilever restoring force. When the voltage difference between the cantilevers and the substrate exceeds the critical voltage, known as the pull-in voltage, ${ }^{52}$ the cantilevers become unstable and pull onto the substrate. Hence, the resonance frequency can be continuously tuned for applied voltages below the pull-in voltage $V_{\text {pull-in }}$.

Figure $4 \mathrm{~b}$ shows a schematic drawing of the cantilever after being released. The radius of curvature of the cantilever is expressed by the following equation: ${ }^{53}$

$$
\frac{1}{R(x)}=\frac{1}{C}\left[\Delta T\left(\alpha_{1}-\alpha_{2}\right)-\left(\frac{1}{E_{1}}+\frac{1}{E_{2}}\right) \frac{2 F_{x}(L-x)}{A\left(t_{1}+t_{2}\right)}\right]
$$

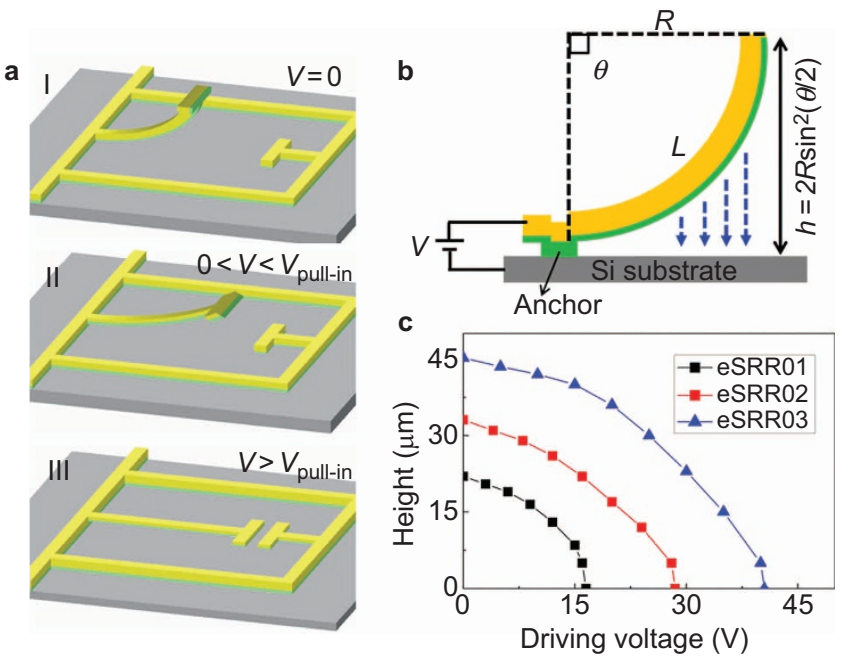

Figure 4 (a) Operation principle of the device: (I) the initial state, (II) the intermediate state and (III) the final state. (b) Schematic drawing of the released cantilever. (c) Relationship between the cantilever height and driving voltage. eSRR, electric split-ring resonator. 
where

$$
\begin{gathered}
C=2 \frac{E_{1} I_{1}+E_{2} I_{2}}{A\left(t_{1}+t_{2}\right)}\left(\frac{1}{E_{1}}+\frac{1}{E_{2}}\right)+\frac{t_{1}+t_{2}}{2} \\
F_{x}=\frac{\varepsilon_{0} A}{2(h-x)^{2}} V^{2} \\
h=2 R \sin ^{2}(\theta / 2)
\end{gathered}
$$

and subscripts 1 and 2 refer to $\mathrm{Al}_{2} \mathrm{O}_{3}$ and $\mathrm{Al}$ materials, respectively; $x$ is the vertical displacement of the released cantilever when a driving voltage is applied; $t$ is the thickness of the thin films $\left(t_{1}=20 \mathrm{~nm}\right.$ and $t_{2}=500 \mathrm{~nm}$ ); $E$ is Young's modulus; $\alpha$ is the thermal expansion coefficient; $I$ is the area moment of inertia; $A$ is the area of the curved beam; and $\Delta T$ is the temperature difference within the fabrication process.

Because the height $h$ of the deformed cantilevers is the most important factor for tuning the resonance frequency of the eSRRs, it is worthy to study the relationship between $h$ and the driving voltage. The cantilever height $h$ is proportional to the driving voltage, i.e., the electrostatic force. Generally, the polygon conformal transformation method, ${ }^{54}$ a typical method for solving complex electromagnetic problems, can be used to obtain an analytical solution of the electrostatic force. Alternatively, a numerical method based on electromagnetic finite element analysis is adopted in this work. Under electrostatic actuation, the height $h$, which varies with the driving voltage, is analyzed by finite element modeling using COMSOL (Burlington, USA) multiphysics software. The finite element simulations were performed using the material parameters listed in Table 1. The calculated relationship between the height of the cantilever and the driving voltage is illustrated in Figure 4c. For eSRR01 (eSRR02/eSRR03), the height $h$ slowly decreases from $h=22(33 / 45)$ to $3(3 / 3) \mu \mathrm{m}$ as the driving voltage changes from 0 to $16(28 / 40) \mathrm{V}$ and then sharply drops to zero when the driving voltage exceeds $16(28 / 40) \mathrm{V}$. This result is due to the 'pull-in's2 instability existing in many electrostatically actuated devices. Hence, the resonance frequency can be continuously tuned in the voltage range from 0 to $V_{\text {pull-in }}$.

\section{Electromagnetic simulation}

Finite-difference time-domain (FDTD) numerical simulations were conducted to calculate the transmission spectra and the electromagnetic field distributions corresponding to the resonance modes with normally incident $\mathrm{THz}$ waves of either e- or o-polarization. Full-field electromagnetic wave simulations were performed using the commercial simulation software Lumerical FDTD with built-in material parameters ( $\mathrm{Al}, \mathrm{Al}_{2} \mathrm{O}_{3}$ and $\mathrm{Si}$ ) from the software's material library. In the simulation, a single unit cell of the metamaterial structures was simulated with periodic boundary conditions employed in axial directions orthogonal to the incident waves. The perfectly matched layers are applied along the propagation of the electromagnetic waves. Plane waves were incident into the unit cell, and the transmission spectrum was monitored with a power monitor placed above the structure. In the meanwhile, frequency profile monitors are used to detect the electric and magnetic fields.

\section{RESULTS AND DISCUSSION}

\section{THz wave of e-polarization}

The transmission of the three proposed metamaterial structures before the cantilever release was first investigated. The measured and simulated transmission spectra of eSRR01, eSRR02 and eSRR03 with epolarization incidence are shown in Figure 5 as circular symbols and solid lines. In this case, the electric field of the incident $\mathrm{THz}$ wave parallel to the split gap-bearing side of the eSRRs excites a circular current due to the charge asymmetry. As expected, there are two distinct resonances in the spectrum of eSRR01, as shown in Figure 5a. These resonances can be understood as plasmonic resonances of the entire structure with increasing order. ${ }^{55}$ The low-frequency resonance is the inductive-capacitive (LC) resonance, where oscillating charges throughout the entire structure are excited due to coupling at the gap. This behavior corresponds to the fundamental plasmonic mode of the metamaterial structure. ${ }^{55}$ The higher-order resonance is dipole-type electrical resonance due to the in-phase dipolar currents along the eSRR components parallel to the electric field of the incident $\mathrm{THz}$ wave. ${ }^{18}$ This electrical resonance is a secondorder plasmon mode of the entire metamaterial structure. ${ }^{55}$ Moreover, three resonances were observed in the spectra of eSRR02 and eSRR03, as shown in Figure 5b and 5c, respectively. The higher frequency dipole resonance undergoes multiple resonance splits as the split gap of the eSRR is moved from the center to the edge of the resonator. The dipole resonance splits into two resonances for eSRR02 (Figure 5b), with the lower resonance at $0.52 \mathrm{THz}$ and the upper resonance at $0.70 \mathrm{THz}$. For eSRR03, the dipole resonance splits into two resonances, with the lower resonance at $0.53 \mathrm{THz}$ and the upper resonance at $0.67 \mathrm{THz}$, as shown in Figure 5c.

To further examine this novel resonance mode, FDTD simulations were performed to calculate the electric and magnetic field strength distribution at the resonance frequencies. Electric and magnetic field strength distributions corresponding to the resonance frequencies labeled as dashed lines in Figure $5 a-5 c$ are shown in Figure $5 d-5 f$. For the LC resonance, the charges strongly accumulate in the split gap of the eSRR, and no significant magnetic field is found along the split gap-bearing side of the eSRRs at the fundamental resonant frequency for any of the resonators, indicating excitation of the LC resonant mode. For the dipole resonance of eSRR01 and the higherorder dipole resonance of eSRR02 and eSRR03, the magnetic field strength is primarily distributed along the eSRR arms and the cantilever parallel to the incident electric field, indicating dipole-type resonance. However, for the lower-order dipole resonance of eSRR02 and eSRR03, the charge is strongly concentrated at the ends of the 'split plate' rather than being concentrated in the split gap, with the magnetic field strongly concentrated on the cantilevers. The asymmetric field distribution indicates that this novel resonance mode results from the structural anisotropy of eSRR02 and eSRR03 caused

\begin{tabular}{|c|c|c|c|c|c|}
\hline Materials & $\begin{array}{l}\text { Young's modulus } \\
\text { (GPa) }\end{array}$ & Poisson's ratio & $\begin{array}{l}\text { Coefficient of thermal expansion } \\
\qquad\left(\times 10^{-6} \mathrm{~K}^{-1}\right)\end{array}$ & $\begin{array}{l}\text { Electrical conductivity } \\
\qquad\left(\times 10^{6} \mathrm{~S} \mathrm{~m}^{-1}\right)\end{array}$ & Density $\left(\mathrm{g} \mathrm{cm}^{-3}\right.$ \\
\hline Si & 169 & 0.3 & 2.568 & 0.025 & 2.33 \\
\hline $\mathrm{Al}$ & 70 & 0.3 & 23.1 & 35.5 & 2.7 \\
\hline
\end{tabular}
by moving the 'split' gap from the resonator's center to its edge.

Table 1 Material properties 


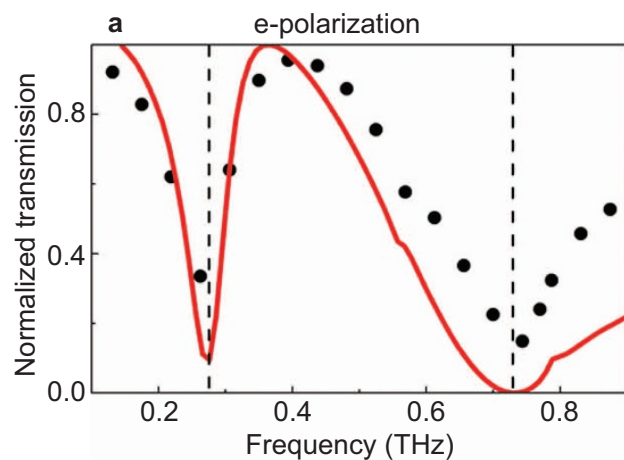

b

e-polarization

c e-polarization
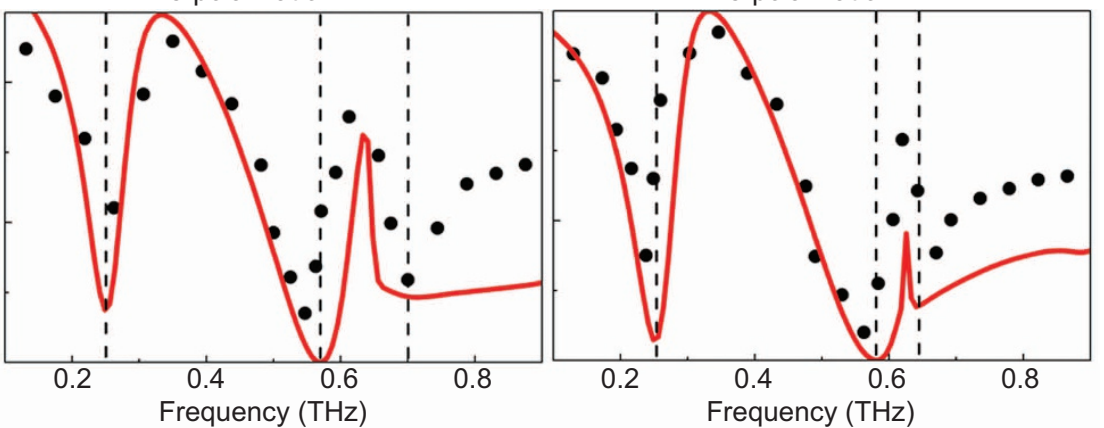

d

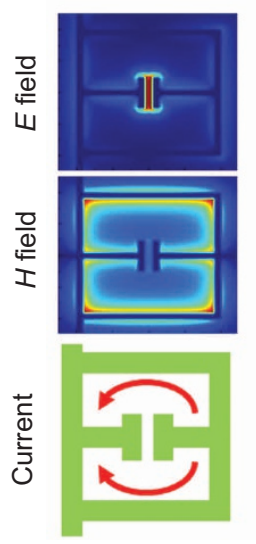

e

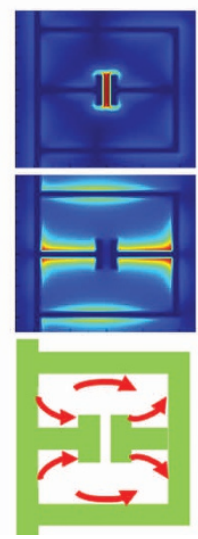

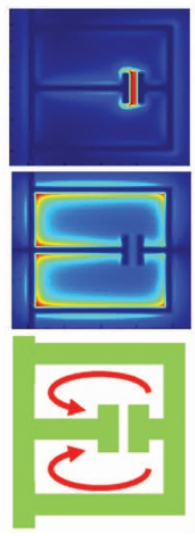

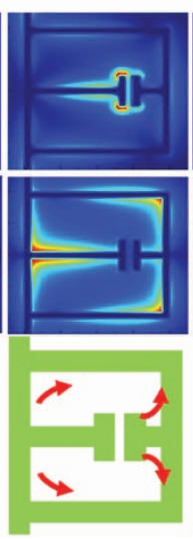

f
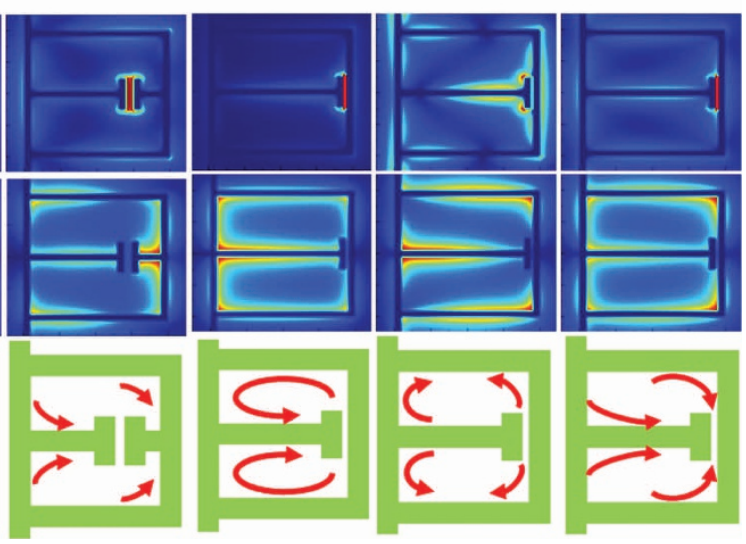

Figure 5 Measured and simulated transmission spectra of non-released (a) eSRR01, (b) eSRR02 and (c) eSRR03 arrays with an incident THz wave of e-polarization. The circular dots and solid lines represent the measured and simulated spectra, respectively. The vertical lines indicate the simulated resonance frequencies. The calculated electric and magnetic field distributions and the induced current (red arrow) of the resonances labeled as vertical dashed lines in a-c are shown for (d) eSRR01, (e) eSRR02 and (f) eSRR03. eSRR, electric split-ring resonator; THz, terahertz.

To investigate the tunability of the proposed metamaterial structures, the transmission of the eSRRs with released bimorph cantilevers was measured. The released bimorph cantilevers used to reconfigure the metamaterial structures were actuated by an electrostatic force due to the DC bias voltage between the chained eSRR patterns and the silicon substrate. As an example, the measured and simulated transmission spectra of the released eSRR02 array at heights of 9, 5 and $0 \mu \mathrm{m}$ are shown in Figure 6. The observed variation of the resonance frequencies is highlighted by the shaded regions, where the transmission spectra are normalized from the minimum to the maximum. It is found that all of the resonances for the three kinds of resonator arrays have a red shift when the cantilevers are actuated down toward the substrate. This behavior can be qualitatively understood by examining the equivalent LC circuit analog of the eSRR. When the released cantilevers bend toward the Si substrate due to the increase in applied voltage, the total length of the eSRR unit cell remains unchanged, i.e., the effective inductance shows little change. However, the capacitance of the equivalent circuit increases as the split gap becomes smaller, resulting in a red shift of the resonance frequencies for the LC resonance. The same resonance shift is observed for the two higher-order modes. These higher-order plasmonic modes are excited because of the non-negligible spatial extension of the structure in comparison to the wavelength. ${ }^{55}$ The effective size of the structure increases as the cantilever bends down toward the substrate. Hence, the resonance frequency decreases as the cantilever bends downward. To investigate the tunability of the released eSRRs, the dependence of the resonance frequencies on the height of the released cantilevers is plotted in

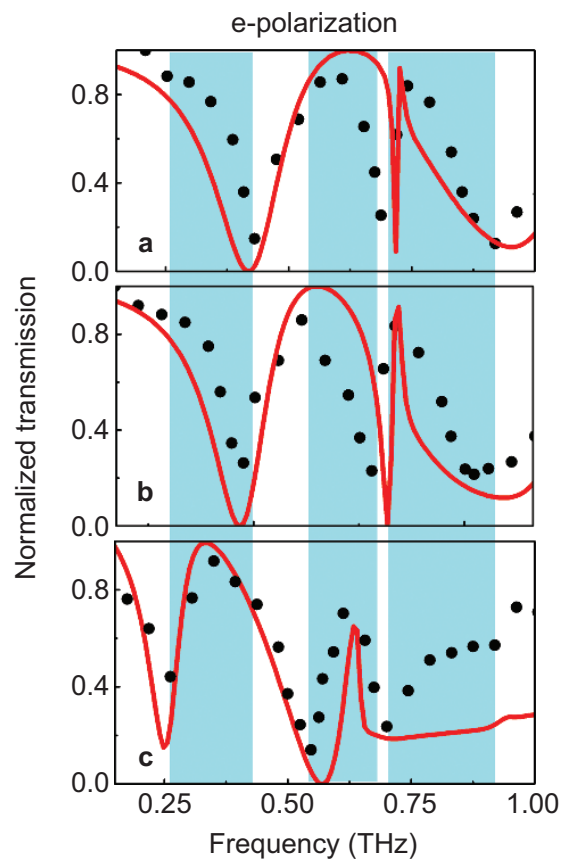

Figure 6 Measured and simulated transmission spectra of the eSRRO2 arrays with an incident $\mathrm{THz}$ wave of e-polarization. The circular symbols and solid lines show the experimentally measured and numerically simulated transmission spectra, respectively. The cantilever height is (a) $9 \mu \mathrm{m}$, (b) $5 \mu \mathrm{m}$ and (c) $0 \mu \mathrm{m}$. The shaded regions represent the shift of the resonance frequencies. eSRR, electric split-ring resonator; THz, terahertz. 

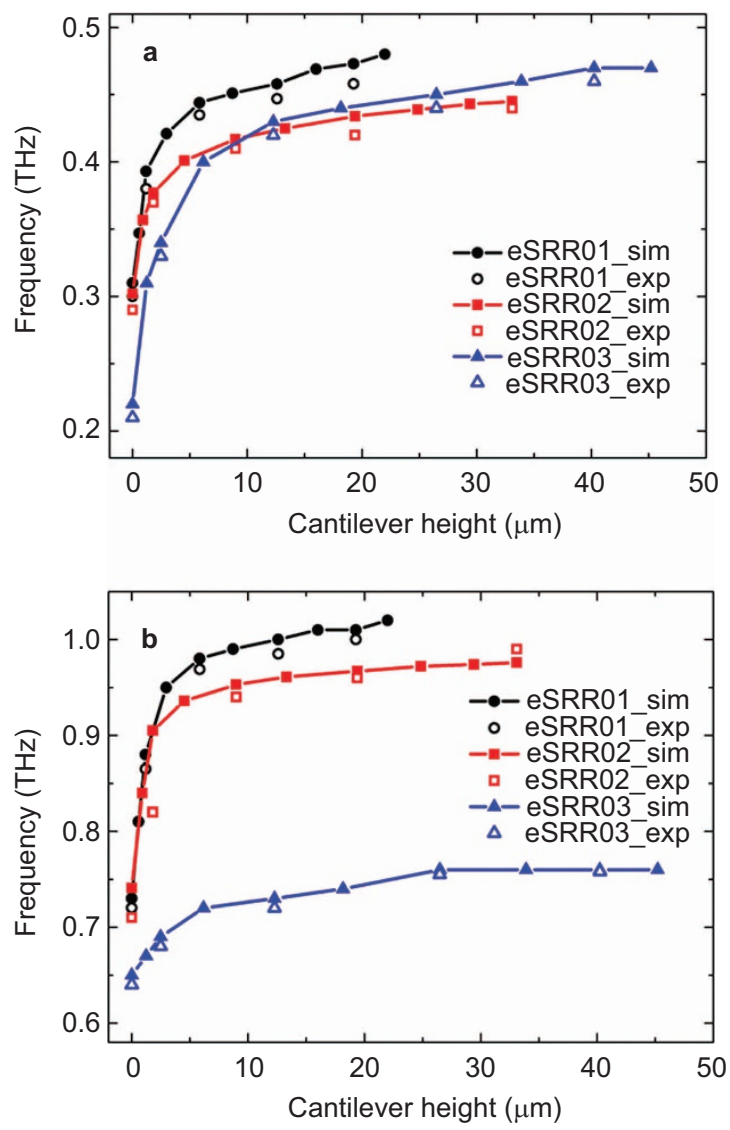

Figure 7 Dependence of the (a) LC and (b) dipole resonant frequencies on cantilever height for the proposed devices. The circular, square and triangle symbols represent the resonance frequencies for eSRR01, eSRRO2 and eSRR03, respectively. The solid and open symbols represent the numerically simulated and experimentally measured values, respectively. eSRR, electric split-ring resonator; LC, inductive-capacitive; $\mathrm{THz}$, terahertz.

Figure 7, with the numerically simulated and experimentally measured values represented by solid and open symbols, respectively. As shown in Figure 7a and 7b, for the eSRR01 structure, the frequency of the LC resonance decreases from 0.45 to $0.31 \mathrm{THz}$, while the frequency decreases from 1.02 to $0.73 \mathrm{THz}$ for the dipole resonance as the height of the released cantilever decreases from 22 to $0 \mu \mathrm{m}$. For the eSRR02 structure, the frequencies of the LC and dipole resonance modes decrease from 0.44 to $0.30 \mathrm{THz}$ and from 0.98 to $0.74 \mathrm{THz}$, respectively, as the height of the released cantilever is decreased from 33 to $0 \mu \mathrm{m}$. For the eSRR03 structure, the frequencies of the LC and dipole resonance modes decrease from 0.47 to $0.22 \mathrm{THz}$ and from 0.76 to $0.65 \mathrm{THz}$ as the height of the released cantilever is decreased from 45 to $0 \mu \mathrm{m}$. By comparing the tunable range of the resonance frequency of these three resonators, it is found that eSRR03 has the largest tunable range of $0.25 \mathrm{THz}$ for the LC resonance, while eSRR01 has the largest tunable range of $0.29 \mathrm{THz}$ for the dipole resonance.

\section{THz wave of o-polarization}

Measured and simulated transmission spectra of the eSRR01, eSRR02 and eSRR03 metamaterial structures with unreleased cantilevers for opolarization incidence are shown in Figure 8 as circular symbols and solid lines, respectively. In this case, the electric field of the incident $\mathrm{THz}$ wave is parallel to the split gap without coupling to the capacitive element. The transmission spectra of the eSRR01, eSRR02 and eSRR03

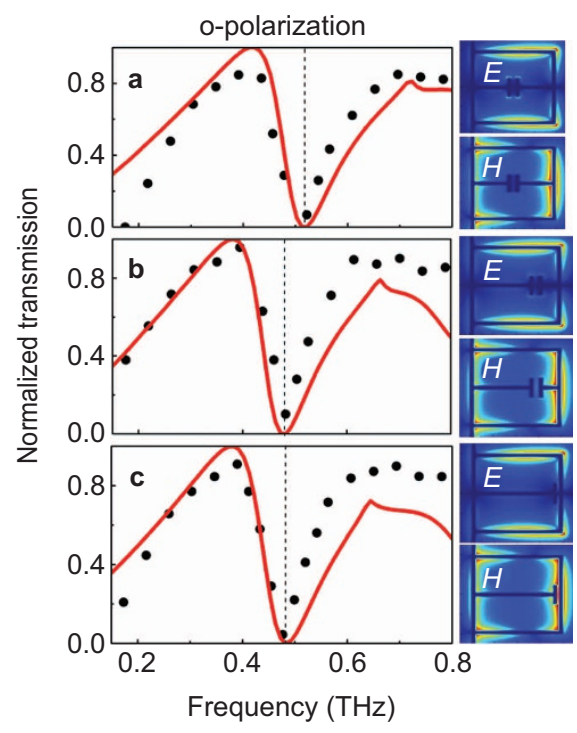

Figure 8 Measured and simulated transmission spectra of non-released (a) eSRR01, (b) eSRR02 and (c) eSRR03 arrays with an incident $\mathrm{THz}$ wave of opolarization. The circular dots and solid lines represent the measured and simulated spectra, respectively. The vertical lines indicate the simulated resonance frequencies. The insets show the calculated electric and magnetic field distributions of the resonances labeled as vertical dashed lines in a-c. eSRR, electric split-ring resonator; $\mathrm{THz}$, terahertz.

metamaterial structures, as shown in Figure 8, exhibit only one resonance. This unique resonance is the result of a superposition of responses of the connecting metal lines. ${ }^{16,56}$ This kind of resonant response results from the dipolar currents in the eSRRs as an analog to the higher-order resonance that occurs when the electric field is perpendicular to the connecting metal lines. ${ }^{16}$ The electric and magnetic field strength distributions calculated for the resonance frequency are shown in Figure 8. The electric and magnetic field strength is high in the eSRR arms rather than along the curved cantilevers. Hence, the movement of the split gap has a trivial effect on the transmission spectra.

Measured and simulated transmission spectra of the released eSRR01, eSRR02 and eSRR03 arrays at cantilever heights of 9, 5 and $0 \mu \mathrm{m}$ are shown in Figure 9. In contrast to the observation for epolarization incidence, the frequency of the Drude-like resonance is unaltered as the height of the released cantilever changes from 9 to $0 \mu \mathrm{m}$ due to the actuation. The different transmission responses due to the e- and o-polarization incidence as a function of cantilever position are primarily due to the resonant features of the eSRR patterns. For e-polarization incidence, the electric field strength is mainly confined in the split gap for the LC and higher-order dipole resonance modes, while the electric field and magnetic field strength is mainly concentrated at the cantilevers for the lower-order resonance mode of the anisotropic eSRRs. However, the cantilevers have a trivial effect on the Drude-like resonance for o-polarization incidence. Therefore, the change in the curved profile of the cantilevers has a significant effect on the observed resonance modes for e-polarization incidence due to the structural change of the split gap.

\section{CONCLUSIONS}

In summary, the design and realization of multiband metamaterials with polarization-dependent transmission behavior are presented. The proposed metamaterials use a MEMS tuning mechanism and 

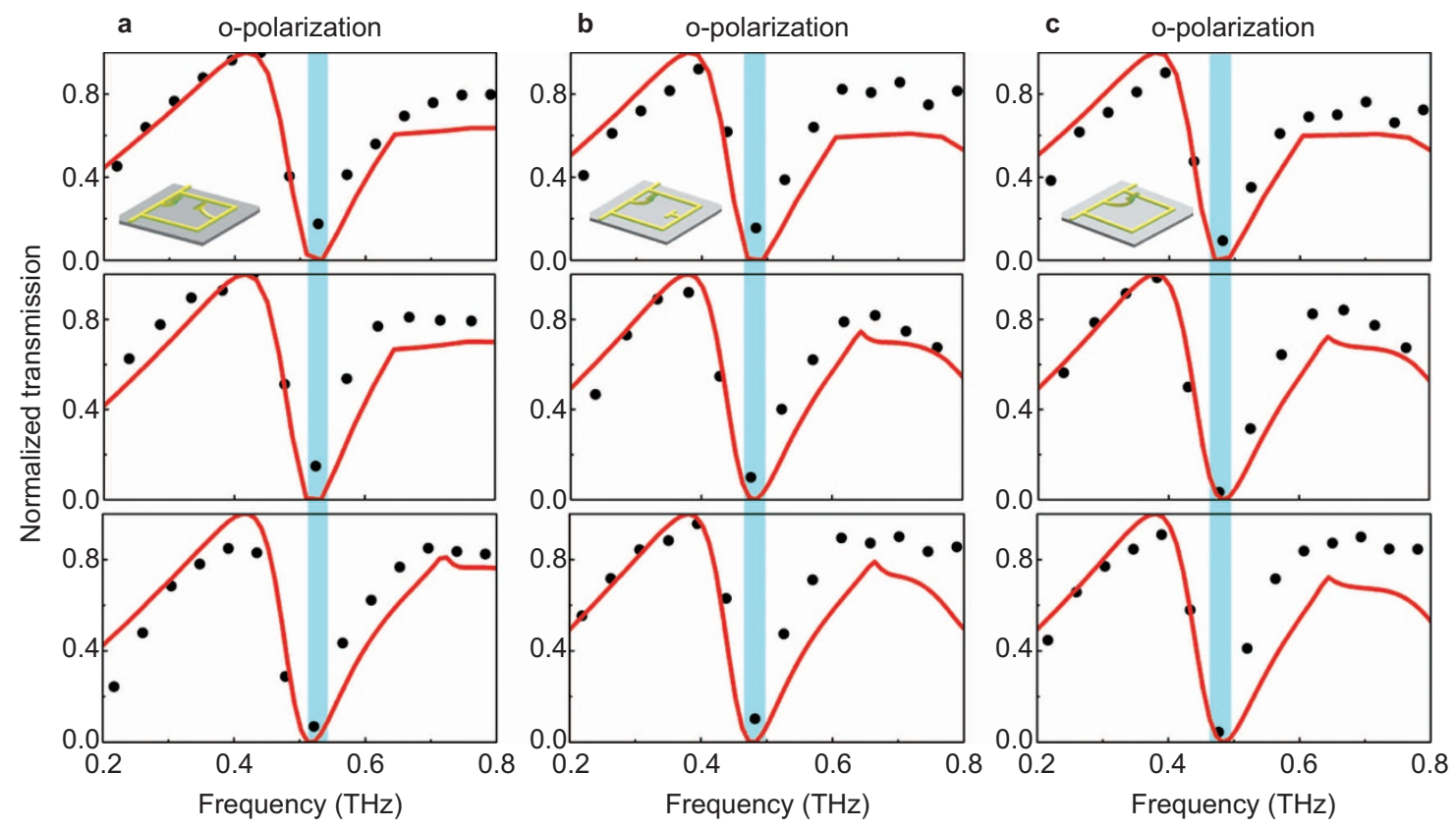

Figure 9 Measured and simulated transmission spectra of the (a) eSRR01, (b) eSRR02 and (c) eSRR03 arrays with an incident THz wave of o-polarization. The circular symbols and solid lines show the experimentally measured and numerically simulated transmission spectra, respectively. The first, second and third rows show the spectra for cantilever heights of 9,5 and $0 \mu \mathrm{m}$, respectively. The shaded regions represent the shift of the resonance frequencies. eSRR, electric split-ring resonator; $\mathrm{THz}$, terahertz.

are fabricated based on a CMOS-compatible process. By varying the position of the split of the electric split-ring resonators, the dipole resonance modes split into two resonances. Together with the fundamental modes of the eSRRs, the split dipole resonances can be tuned by bending the incorporated cantilevers out of plane when the electric field of the incident $\mathrm{THz}$ wave is perpendicular to the split gap of the eSRRs. In contrast, the resonance frequencies are not tunable when the electric field of the incident $\mathrm{THz}$ wave is parallel to the split gap of the eSRRs. Hence, the tunability of the proposed structures is dependent on the polarization of the incident $\mathrm{THz}$ waves. Numerical simulations reproduce the experimentally measured results with reasonable agreement. The observed polarization-dependent properties may be desirable for potential applications in the development of polarization-sensitive $\mathrm{THz}$ polarimetric devices. Furthermore, the approach presented here is not limited to $\mathrm{THz}$ frequencies but can be readily extended over the entire electromagnetic spectrum.

\section{ACKNOWLEDGMENTS}

This work was supported by MOE2012-T2-2-154 (Monolithic Integrated Si/ AIN Nanophotonics Platform for Optical NEMS and OEICs) under WBS No. R-263-000-A59-112.

1 Schurig D, Mock JJ, Justice BJ, Cummer SA, Pendry JB et al. Metamateria electromagnetic cloak at microwave frequencies. Science 2006; 314: 977-980.

2 Shelby RA, Smith DR, Schultz S. Experimental verification of a negative index of refraction. Science 2001; 292: 77-79.

3 Smith DR, Mock JJ, Starr AF, Schurig D. Gradient index metamaterials. Phys Rev E 2005; 71: 036609 .

4 Smith DR, Padilla WJ, Vier DC, Nemat-Nasser SC, Schultz S. Composite medium with simultaneously negative permeability and permittivity. Phys Rev Lett 2000; 84: 4184-4187.

5 Linden S, Enkrich C, Wegener M, Zhou J, Koschny T et al. Magnetic response of metamaterials at 100 terahertz. Science 2004; 306: 1351-1353.
6 O'Hara JF, Smirnova E, Chen HT, Taylor AJ, Averitt RD et al. Properties of planar electric metamaterials for novel terahertz applications. J Nanoelectron Optoelectron 2007; 2: 90-95.

7 Padilla WJ, Aronsson MT, Highstrete C, Lee M, Taylor AJ et al. Electrically resonant terahertz metamaterials: theoretical and experimental investigations. Phys $\operatorname{Rev} B$ 2007; 75: 041102(R)

8 Soukoulis CM, Koschny T, Zhou J, Kafesaki M, Economou EN. Magnetic response of split ring resonators at terahertz frequencies. Phys Status Solidi B 2007; 244: 11811187.

9 Yen TJ, Padilla WJ, Fang N, Vier DC, Smith DR et al. Terahertz magnetic response from artificial materials. Science 2004; 303: 1494-1496.

10 Landy NI, Sajuyigbe S, Mock JJ, Smith DR, Padilla WJ. Perfect metamaterial absorber. Phys Rev Lett 2008; 100: 207402.

11 Liu R, Degiron A, Mock JJ, Smith DR. Negative index material composed of electric and magnetic resonators. Appl Phys Lett 2007; 90: 263504.

12 Liu R, Cui TJ, Zhao B, Lin XQ, Ma HF et al. Resonant crystal band gap metamaterials in the microwave regime and their exotic amplification of evanescent waves. Appl Phys Lett 2007; 90: 091912.

13 Bingham CM, Tao H, Liu X, Averitt RD, Zhang $\mathrm{X}$ et al. Planar wallpaper group metamaterials for novel terahertz applications. Opt Express 2008; 16: 1856518575.

14 Chen HT, O'Hara JF, Taylor AJ, Averitt RD, Highstrete C et al. Complementary planar terahertz metamaterials. Opt Express 2007; 15: 1084-1095.

15 Chen Z, Mohsen R, Gong Y, Chong CT, Hong M. Realization of variable threedimensional terahertz metamaterial tubes for passive resonance tunability. Adv Mater 2012; 24: OP143-OP147.

16 Chen HT, Padilla WJ, Zide JM, Gossard AC, Taylor AJ et al. Active terahertz metamaterial devices. Nature 2006; 444: 597-600.

17 Chen HT, O'Hara JF, Azad AK, Taylor AJ, Averitt RD et al. Experimental demonstration of frequency-agile terahertz metamaterials. Nat Photonics 2008; 2: 295-298.

18 Padilla WJ, Taylor AJ, Highstrete C, Lee M, Averitt RD. Dynamical electric and magnetic metamaterial response at terahertz frequencies. Phys Rev Lett 2006; 96 : 107401.

19 Han J, Lakhtakia A, Qiu CW. Terahertz metamaterials with semiconductor split-ring resonators for magnetostatic tunability. Opt Express 2008; 16: 14390-14396.

20 Chen HT, Lu H, Azad AK, Averitt RD, Gossard AC et al. Electronic control of extraordinary terahertz transmission through subwavelength metal hole arrays. Opt Express 2008; 16: 7641-7648.

21 Chen HT, Palit S, Tyler T, Bingham CM, Zide JM et al. Hybrid metamaterials enable fast electrical modulation of freely propagating terahertz waves. Appl Phys Lett 2008; 93: 091117.

22 Busch K, John S. Liquid-crystal photonic-band-gap materials: the tunable electromagnetic vacuum. Phys Rev Lett 1999; 83: 967-970.

23 Kahn FJ. Electric-field-induced orientational deformation of nematic liquid crystals: tunable birefringence. Appl Phys Lett 1972; 20: 199-201. 
24 Liu Z, Huang CY, Liu H, Zhang X, Lee C. Resonance enhancement of terahertz metamaterials by liquid crystals/indium tin oxide interfaces. Opt Express 2013; 21: 6519-6525.

25 Chen HT, O'Hara JF, Azad AK, Taylor AJ. Manipulation of terahertz radiation using metamaterials. Laser Photon Rev 2011; 5: 513-533.

26 Chen HT, Yang H, Singh R, O'Hara JF, Azad AK et al. Tuning the resonance in hightemperature superconducting terahertz metamaterials. Phys Rev Lett 2010; 105: 247402.

27 Singh R, Xiong J, Azad AK, Yang H, Trugman SA et al. Optical tuning and ultrafast dynamics of high-temperature superconducting terahertz metamaterials. Nanophotonics 2012; 1: 117-123.

28 Singh R, Azad AK, Jia QX, Taylor AJ, Chen HT. Thermal tunability in terahertz metamaterials fabricated on strontium titanate single-crystal substrates. Opt Lett 2011; 36: 1230-1232.

29 Chen HT, Padilla WJ, Cich MJ, Azad AK, Averitt RD et al. A metamaterial solid-state terahertz phase modulator. Nat Photonics 2009; 3: 148-151.

30 Ekmekci E, Topalli K, Akin T, Turhan-Sayan G. A tunable multi-band metamaterial design using micro-split SRR structures. Opt Express 2009; 17: 16046-16058.

$31 \mathrm{He} \mathrm{G}$, Wu RX, Poo Y, Chen P. Magnetically tunable double-negative material composed of ferrite-dielectric and metallic mesh. J Appl Phys 2010; 107: 093522.

32 Chan WL, Chen HT, Taylor AJ, Brener I, Cich MJ et al. A spatial light modulator for terahertz beams. App/ Phys Lett 2009; 94: 213511.

33 Yuan Y, He J, Liu J, Yao J. Proposal of an electrically controlled terahertz switch based on liquid-crystal-filled dual-metallic grating structures. App/ Opt 2010; 49: 6092-6097.

34 Huang WX, Yin XG, Huang CP, Wang QJ, Miao TF et al. Optical switching of a metamaterial by temperature controlling. App/ Phys Lett 2010; 96: 261908.

35 Dicken MJ, Aydin K, Pryce IM, Sweatlock LA, Boyd EM et al. Frequency tunable nearinfrared metamaterials based on $\mathrm{VO}_{2}$ phase transition. Opt Express 2009; 17 : 18330-18339.

36 Jin B, Zhang C, Engelbrecht S, Pimenov A, Wu J et al. Low loss and magnetic field-tunable superconducting terahertz metamaterial. Opt Express 2010; 18: 17504-17509.

37 Lapine M, Powell D, Gorkunov M, Shadrivov I, Marqués R et al. Structural tunability in metamaterials. Appl Phys Lett 2009; 95: 084105.

38 Tao H, Strikwerda AC, Fan K, Padilla WJ, Zhang X et al. Reconfigurable Terahertz Metamaterials. Phys Rev Lett 2009; 103: 147401.

39 Hand T, Cummer S. Characterization of tunable metamaterial elements using MEMS switches. IEEE Antennas Wirel Propag Lett 2007; 6: 401-404.

40 Zhu WM, Liu AQ, Bourouina T, Tsai DP, Teng JH et al. Microelectromechanical Maltese-cross metamaterial with tunable terahertz anisotropy. Nat Commun 2012; 3: 1274.

41 Zhang W, Zhu WM, Cai H, Tsai ML, Lo GQ et al. Resonance switchable metamaterials using MEMS fabrications. IEEE J Sel Top Quant Electron 2013; 19: 4700306.

42 Zhu WM, Liu AQ, Zhang XM, Tsai DP, Bourouina T et al. Switchable magnetic metamaterials using micromachining processes. Adv Mater 2011; 23: 1792-1796.
43 Tao H, Strikwerda AC, Fan K, Padilla WJ, Zhang X et al. MEMS based structurally tunable metamaterials at terahertz frequencies. J Infrared Milli Terahz Waves 2011; 32: $580-595$.

44 Ozbey B, Aktas O. Continuously tunable terahertz metamaterial employing magnetically actuated cantilevers. Opt Express 2011; 19: 5741-5752.

45 Lin YS, Ma FS, Lee C. Three-dimensional movable metamaterials using electric splitring resonators. Opt Lett 2013; 38: 3126-3128.

46 Ma FS, Qian Y, Lin YS, Liu H, Zhang X et al. Polarization-sensitive microelectromechanical systems based tunable terahertz metamaterials using three dimensional electric splitring resonator arrays. Appl Phys Lett 2013; 102: 161912.

47 Lin YS, Qian Y, Ma FS, Liu Z, Kropelnicki P et al. Development of stress-induced curved actuators for a tunable $\mathrm{THz}$ filter based on double split-ring resonators. Appl Phys Lett 2013; 102: 111908.

48 Kwon DH, Werner DH, Kildishev AV, Shalaev VM. Near-infrared metamaterials with dual-band negative-index characteristics. Opt Express 2007; 15: 1647-1652.

49 Yuan Y, Bingham C, Tyler T, Palit S, Hand TH et al. Dual-band planar electric metamaterial in the terahertz regime. Opt Express 2008; 16: 9746-9752.

50 Zhu C, Ma JJ, Li L, Liang $\mathrm{CH}$. Multiresonant metamaterial based on asymmetric triangular electromagnetic resonators. IEEE Antennas Wirel Propag Lett 2010; 9: 99-102.

51 Chen CC, Hsiao CT, Sun S, Yang KY, Wu PC et al. Fabrication of three dimensional split ring resonators by stress-driven assembly method. Opt Express 2012; 20: 9415 9420.

52 Zhang Y, Zhao YP. Numerical and analytical study on the pull-in instability of microstructure under electrostatic loading. Sens Actuators A Phys 2006; 127: 366-380.

53 Shavezipur M, Guo W, Carraro C, Maboudian R. Characterization of adhesion force in MEMS at high temperature using thermally actuated microstructures. J Microelectromech Syst 2012; 21: 541-548.

54 Gibbs WJ. Conformal Transformations in Electrical Engineering. London: Chapman and Hall; 1958.

55 Rockstuhl C, Lederer F, Etrich C, Zentgraf T, Kuhl J et al. On the reinterpretation of resonances in split-ring-resonators at normal incidence. Opt Express 2006; 14: 8827-8836.

56 Pendry JB, Holden AJ, Stewart WJ, Youngs I. Extremely low frequency plasmons in metallic mesostructures. Phys Rev Lett 1996; 76: 4773-4776.

\section{(c) (i)(5) This work is licensed under a Creative Commons Attribution-}

(c) ${ }_{B Y}$ NC SA NonCommercial-ShareAlike 3.0 Unported License. The images or other third party material in this article are included in the article's Creative Commons license, unless indicated otherwise in the credit line; if the material is not included under the Creative

Commons license, users will need to obtain permission from the license holder to reproduce the material. To view a copy of this license, visit http://creativecommons.org/licenses/by-nc-sa/3.0/ 